\title{
Hand, Foot and Mouth Disease (HFMD): Prevalence and its Spatial Relationship with Vaccine Refusal Cases in Terengganu, Malaysia
}

\author{
Said, Z. M., ${ }^{1}$ Chen, X. W., ${ }^{2}$ Aziz, W. A. A. A., ${ }^{3}$ Wahab, A. ${ }^{4}$ and Musa, K. I. ${ }^{*}$ \\ ${ }^{1}$ Department of Community Medicine, School of Medical Sciences, Health Campus, Universiti Sains \\ Malaysia, He alth Campus, 16150 Kubang Kerian, Kelantan, Malaysia \\ E-mail: zarudinmatsaid@gmail.com,drkamarul@usm.my \\ ${ }^{2}$ Department of Public Health Medicine, Faculty of Medicine, Universiti Teknologi MARA, Sungai Buloh \\ Campus, Jalan Hospital, 47000 Sungai Buloh, Selangor, Malaysia, E-mail: drchenxw@uitm.edu.my \\ ${ }^{3}$ Kota Bharu District Health Office, Jalan Doktor, Bandar Kota Bharu, 15000 Kota Bharu, Kelantan, \\ Malaysia, E-mail: wiraalfatah@gmail.com \\ ${ }^{4}$ Communicable Disease Control and Prevention Unit, Terengganu State Health Department, 20920 Kuala \\ Terengganu, Terengganu, Malaysia, E-mail: azmani@moh.gov.my \\ *Correspondence Author
}

DOI: https://doi.org/10.52939/ijg.v17i5.2001

\begin{abstract}
Hand, foot and mouth disease (HFMD) is a global public health problem with pandemic potential. The progressive increment of HFMD cases in Malaysia needs further investigation to identify the pattern of disease spread, including its proximity to vaccine refusal. We sought to estimate the prevalence of HFMD in Terengganu and determine the spatial relationship between HFMD and vaccine refusal cases. This study employed data from the national electronic communicable disease notification system and vaccine refusal database maintained by the Communicable Disease Control (CDC) Unit and Maternal and Child Health Care (MCH) Unit. Data from all cases recorded in 2016 were provided by the Terengganu State Health Department, Malaysia. The number of HFMD cases for each district was estimated using the points-inpolygons function in $R$ software. The spatial relationship between HFMD cases and vaccine refusal cases was tested using the cross K-function test. A total of 811 HFMD cases was notified in 2016, with the overall prevalence at 80.2 cases per 100,000 population. Among all districts in Terengganu, the prevalence of HFMD ranged from 19.2 to 230.9 cases per 100,000 population, with the cases highly concentrated in three districts: Kuala Terengganu, Marang, and Dungun. There was evidence of a spatial cluster of HFMD cases based on the Nearest Neighbour Index, $r=0.27$ (p-value $<0.01$ ). Moreover, the locations of HFMD cases were statistically and closely related to the areas of vaccine refusal cases (cross $K$ test, $p$-value $<0.010$ ). The prevalence of HFMD from year to year was high. HFMD cases and vaccine refusal cases formed clusters in the districts with a high-density population. The proximity of HFMD cases and vaccine refusal cases in Terengganu warrants further investigation.
\end{abstract}

\section{Introduction}

Hand, foot, and mouth disease (HFMD) is a common childhood illness caused by various enteroviruses (Xing et al., 2014). A person is most contagious during the first week of the disease, and most of the outbreaks are often found in nurseries, playgroups, schools, and households where young children have lots of close contact with one another (Chang et al., 2011). Outbreaks of HFMD have been reported in countries of the Western Pacific Region and South-East Asia, including Malaysia, and the cases are increasing every year (Charoenchokpanit and Pumpaibool, 2013). The average incidence rate of HFMD in Malaysia for the year 2000 to 2008 was 25.0 ranged from 1.5 to 60.6 per 100,000 population (Chang et al., 2011). Furthermore, local statistics reported an increment of $347.33 \%$ in the HFMD cases, from 7,002 to 31,322 cases in 2011 and 2014, respectively, highlighting the rapid increment in most of the states in Malaysia (MOH, 2016). However, from our extensive search, no study has yielded the latest prevalence of HFMD in Malaysia. It was reported that the population density significantly affects the risk for HFMD events. This includes the population of the states or districts and 
the population density among the household (Huang et al., 2014). The household setting, schools, and communities play essential roles in transmitting HFMD (Chang et al., 2004). The climate factors and the child population density, population immunity, and environmental hygiene contribute to the occurrence, transmission, and spread of HFMD (Hu et al., 2012).

Furthermore, a recent nationwide study highlighted that the overall immunisation coverage of $86.4 \%$ in Malaysia was unsatisfactory (Lim et al., 2017), and vaccine refusal was one of the determinants of low immunisation coverage in Malaysia (Ahmad et al., 2017). Vaccine refusal refers to the individuals who had refused some or all vaccines scheduled in the Malaysia National Immunization Programme (Lim et al., 2016). There is an increasing number of vaccine refusal, and hesitancy worldwide, including Malaysia, wherein the number of parents who refused to get their children vaccinated has almost increased three-fold, from 637 cases in 2013 to 1054 cases in the middle of the year 2015 (Ahmad et al., 2017). Terengganu reported the second-highest number of unimmunised children (233 cases) in Malaysia (MOH, 2018).

The utilisation of a geographic information system (GIS) has not been extensively used in public health research (including that of HFMD) in Malaysia. We found only a single study on HFMD using GIS in 2012, in which they gathered data from 11 districts of Sarawak (Sham et al., 2014). Their findings on the spatial distribution and analyses were used to facilitate public health team responses upon HFMD outbreaks (Sham et al., 2014).

Therefore, we aimed to use the national surveillance data from the Ministry of Health Malaysia to estimate the overall, sex-, age-, and district-specific prevalence of HFMD cases in Terengganu. We also sought to estimate the density of HFMD and vaccine refusal case distribution, subsequently determine their spatial relationship.

\section{Methods}

\subsection{Study Area}

Terengganu is a state of Malaysia located in the East of Peninsular Malaysia and bounded to Kelantan in the north and northwest; the South China Sea in the east; and Pahang in the south and southwest. The state has a total area of $12,959 \mathrm{~km}^{2}$ with a total population of 1,011,363 based on the Population and Housing Census 2010, Department of Statistics Malaysia (Brinkhoff, 2018). Terengganu has eight administrative districts, including Besut, Dungun, Hulu Terengganu, Kemaman, Kuala Nerus, Kuala Terengganu, Marang and Setiu.
The ethnic composition of Terengganu shows that $97 \%$ of the population is Malay and Bumiputera, $2.6 \%$ is Chinese, $0.2 \%$ is Indian, and $0.1 \%$ represents the others. The age distribution shows that the $0-14$ years-old group comprises $32.3 \%$ of the total population. This is followed by the 15-64 years-old group $(62.8 \%)$ and the elderly (aged 65 years and above) comprises $4.9 \%$ of the total population (Brinkhoff, 2018).

\subsection{Data Sources and Case Ascertainment}

In this study, we employed two data sources, 1) the notified HFMD cases made available from the Malaysia Ministry of Health through the national electronic communicable disease notification system [Communicable Diseases Control Information System (CDCIS) E-Notification Version 2011.1], and 2) the vaccine refusal registry from the Maternal and Child Health $(\mathrm{MCH})$ Unit database governed by the Child Division of Terengganu State Health Department. The Terengganu State Health Department notified and registered both types of data from January to December 2016. We excluded missing coordinates and imported HFMD cases, such as cases that lived outside Terengganu.

The CDCIS system contains HFMD-related data and the coordinates that were referring to the residence of the cases and were geocoded during active case investigation. While the vaccine refusal cases were documented once the cases were reported from the health clinic or district health office by $\mathrm{MCH}$ Unit. Their residential addresses were geocoded during the routine immunisation schedule or home visit. Both the coordinates of the notified HFMD cases and vaccine refusal cases were converted into the format of the projected coordinate system; the Kertau Rectified Skewed Orthomorphic (RSO) Malaya (EPSG:3168). Apart from that, we also obtained the census tract derived from the latest nationwide census and contained the aggregated demographic data for the state and districts of Terengganu (DOSM, 2019).

\subsection{Statistical Analysis}

We estimated the overall prevalence of HFMD cases in Terengganu 2016 by dividing the total number of confirmed HFMD cases by the total population size of the Terengganu. First, data of HFMD cases in the Microsoft Excel format were converted into the ESRI shapefile (.shp) format using Quantum Geographic Information System (QGIS) (Lacaze et al., 2018). Then, we calculated the prevalence according to sex, age, and district. Subsequently, we performed a spatial distribution analysis using QGIS for heat map analysis, a graphical representation of point data showing different colours to represent 
areas with varying concentrations of points and trends (Yeap and Uy, 2014). Next, we used the point pattern analysis to examine the spatial spread (clustered, dispersed, or random) based on the Nearest Neighbour Index (NNI) analysis (Lai et al., 2008). The NNI measures the spatial distribution from zero (clustered pattern) to one (randomly dispersed pattern) to 2.15 (regularly dispersed /uniform pattern) (Lai et al., 2008). The spatial relationship between the HFMD and vaccine refusal cases was assessed using Ripley's K-function (a.k.a cross K-function) analysis from the 'spastat' package in R Software Version 3.5.1 (Baddeley and Turner, 2005 and Core and Team, 2013). The Kfunction was calculated in a bivariate form between both cases and counted the number of neighbouring HFMD cases found within a given distance of each vaccine refusal case. Then, the significant level of the function was compared to a confidence limit for random distribution created using Monte Carlo simulations. The upper and lower bounds of these permutations were plotted together with the observed difference function. Any deviation above the envelope formed by the upper and lower bounds indicates significant clustering of cases relative to non-cases/controls (referring to vaccine refusal cases) (Lawson, 2018).

\section{Results}

We obtained records from 811 HFMD notified cases to the CDCIS in 2016. The mean and standard deviation (SD) ages of HFMD were 2.48, 3.36 years, respectively. Table 1 showed the characteristics of the study subjects, the distributions of HFMD cases based on sex, age categories, and district, with the corresponding prevalence rate per 100,000 populations. Almost all patients were from the Malay population with $97.6 \%(792 / 811)$, while $2.4 \%(19 / 811)$ were from Chinese, and $(1.6 \%$, 13/811) from Indian ethnic groups. In Figure 1, we showed the age distribution of HFMD cases, wherein the highest age group contributing was oneyears $(34.2 \%, 277 / 811)$, and the percentage decreases with the increment of age until the age of 15 years. The cases aged less than one year were $8.8 \%(71 / 811)$ of the total notified cases in 2016 . The majority of HFMD cases, $89.6 \%$ (727/811), were classified as single/sporadic cases, and only $10.4 \%(84 / 811)$ were classified as clusters or outbreaks in Terengganu. A similar trend was found for both single and cluster cases across the epidemiological week in 2016.

\subsection{Geographical Distribution of HFMD Cases and Vaccine Refusal Cases in Terengganu \\ Based on the distribution pattern (see Figure 2), it was shown that HFMD cases were located in the central region or urban area of every district, including Kuala Terengganu, Marang, and Dungun. However, in Setiu, Hulu Terengganu, part of Dungun, and Kemaman, the HFMD cases also were randomly distributed over the district region.}

Table 1: The overall, age-specific, sex-specific, and district-specific prevalence of HFMD cases

\begin{tabular}{|l|c|c|c|}
\hline Variables & $\mathbf{n ~ ( \% )}$ & Prevalence* $^{*}$ & Total Population \\
\hline Overall & 811 & 80.2 & $1,011,363$ \\
\hline Sex & & & \\
\hline Male & $460(56.7)$ & 89.2 & 515,579 \\
\hline Female & $351(43.3)$ & 70.8 & 495,784 \\
\hline Age-groups & & & \\
\hline $0-4$ years & $724(89.3)$ & 760.6 & 95,189 \\
\hline $5-9$ years & $73(9.0)$ & 63.7 & 114,528 \\
\hline $10-14$ years & $14(1.7)$ & 12.0 & 116,803 \\
\hline Districts & & & \\
\hline Besut & $72(8.9)$ & 52.7 & 136,563 \\
\hline Dungun & $117(14.4)$ & 78.1 & 149,851 \\
\hline Hulu Terengganu & $59(7.3)$ & 83.3 & 70,800 \\
\hline Kemaman & $32(3.9)$ & 19.2 & 166,750 \\
\hline K.Terengganu / K. Nerus & $276(34.0)$ & 81.8 & 337,553 \\
\hline Marang & $129(15.9)$ & 135.4 & 95,283 \\
\hline Setiu & $126(15.5)$ & 230.9 & 54,563 \\
\hline
\end{tabular}




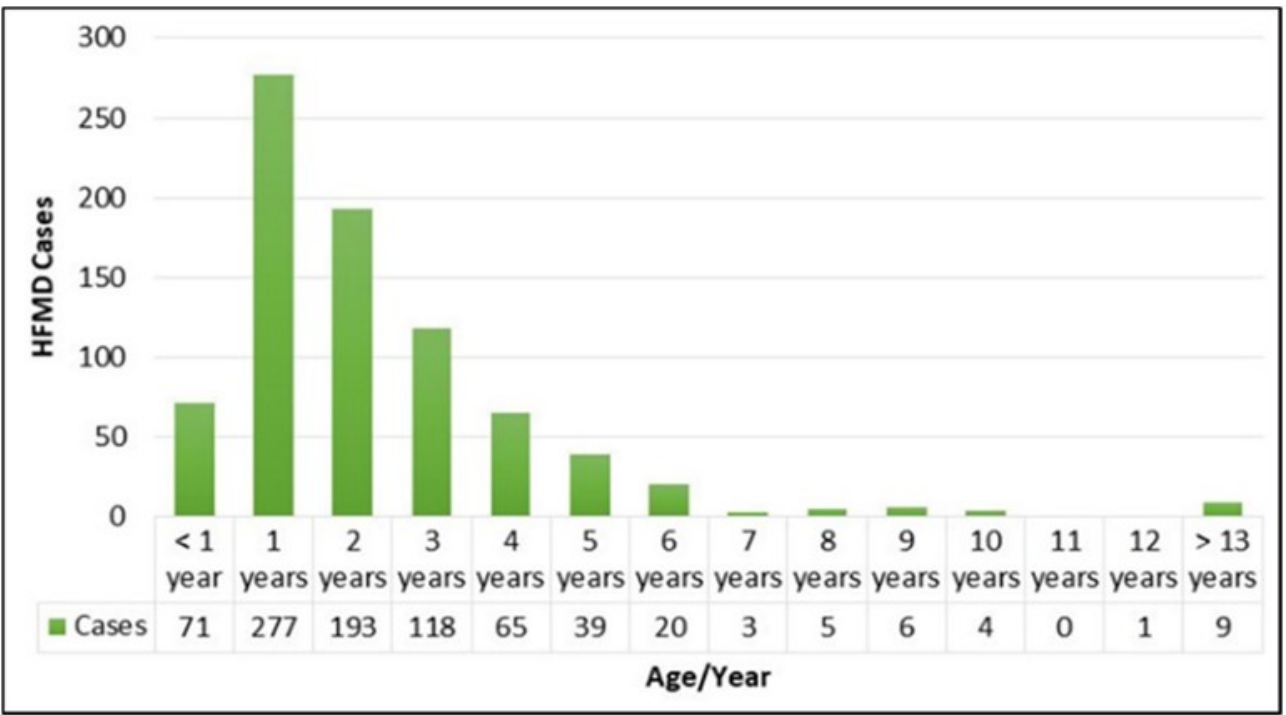

Figure 1: Distributions of HFMD cases in Terengganu in 2016 by age

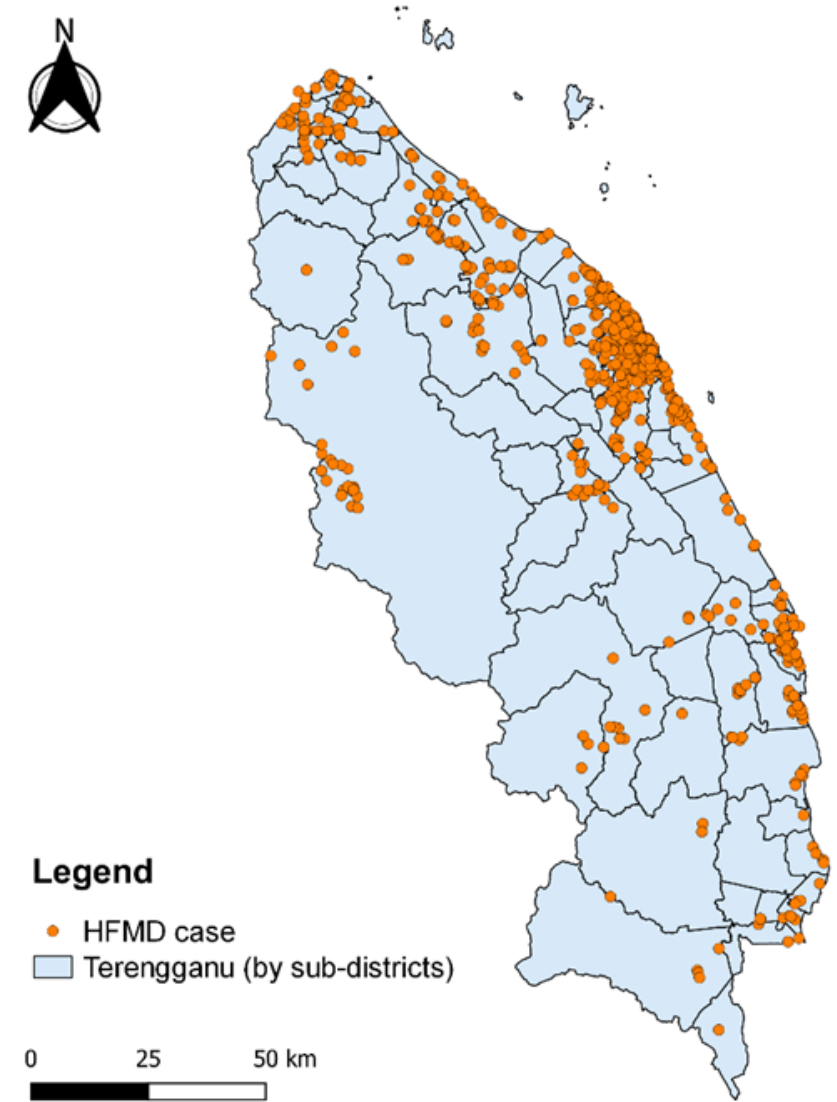

Figure 2: Geographical distribution of HFMD cases in Terengganu

There were 281 vaccine refusal cases registered in Terengganu for the year 2016. The highest prevalence of vaccine refusal was in Kuala Terengganu $(26.7 \%, 75 / 281)$, followed by Dungun (17.8\%, 50/281), Kemaman (16\%, 45/281), Marang
(13.2\%, 37/281), and Kuala Nerus (12.8\%, 36/281). Other districts were contributing to less than $10 \%$ of the total vaccine refusal cases - Hulu Terengganu district $(7.5 \%, 21 / 281)$, Setiu district $(3.9 \%, 11 / 281)$, and Besut district $(2.1 \%, 6 / 281)$. Figure 3 showed 
the geographical distribution pattern of the vaccine refusal cases, wherein the cases were primarily concentrated over the high-density population areas, such as in Kuala Terengganu, Marang, Dungun, and Kemaman. The cases appeared to concentrate in the central region or urban area. However, in Besut and Setiu, the distribution was less centralised and randomly located all over the district region.

\subsection{The Cluster of HFMD and Vaccine Refusal Cases in Terengganu}

The NNI value for HFMD cases was 0.27 indicated clusters among the cases, which can be due to the large population density in those areas rather than the actual infection dynamics. The vaccine refusal cases also formed clusters among the cases with NNI value of 0.23 . The smaller the value of NNI, the more significant the clusters would be. Table 2 showed that both the results were significant with a $p$-value $<0.01$.

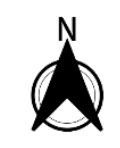

\section{Legend}

- Vaccine refusal case Terengganu (by sub-districts)

0
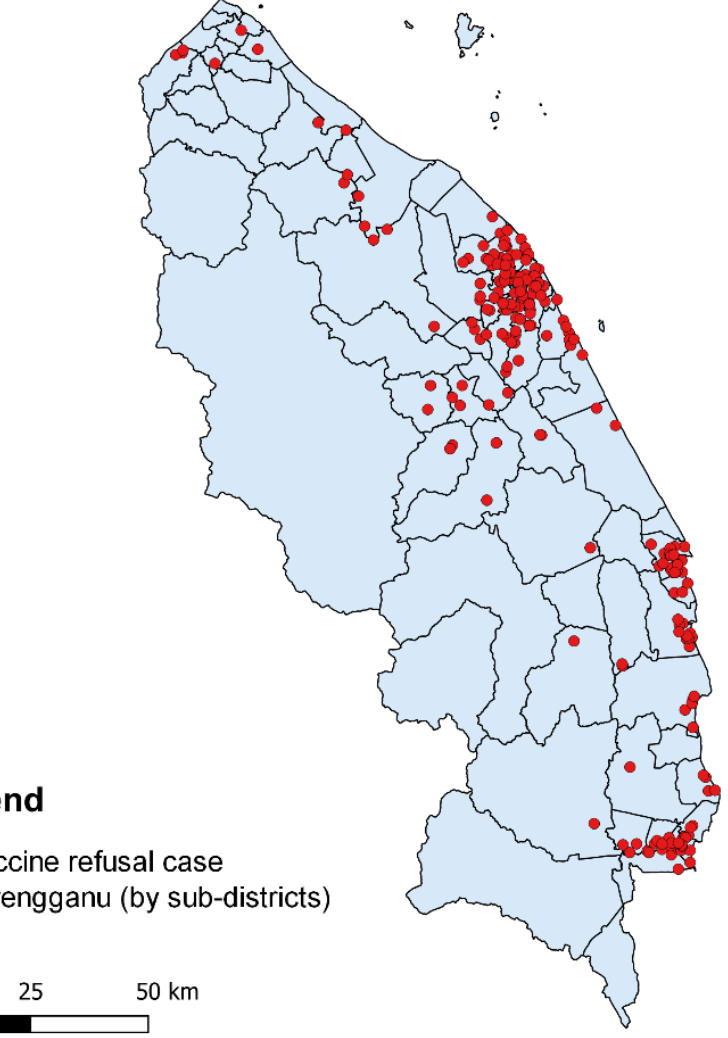

Figure 3: Geographical distribution of vaccine refusal cases in Terengganu

Table 2: Nearest Neighbouring Index (NNI) for HFMD and vaccine refusal cases in Terengganu

\begin{tabular}{|l|l|c|c|c|c|l|}
\hline \multirow{2}{*}{ Variable } & \multirow{2}{*}{$\mathbf{n}$} & \multicolumn{2}{|c|}{ Observed Expected } & \multirow{2}{*}{ NNI } & \multirow{z}{*}{ score } & \multirow{2}{*}{$\boldsymbol{p}$-value* } \\
\cline { 3 - 7 } & & \multicolumn{2}{|c|}{ Mean Distance } & & \\
\hline HFMD & 811 & 691.23 & 2695.04 & 0.27 & -40.51 & $<\mathbf{0 . 0 1}$ \\
\hline Vaccine Refusal & 281 & 979.26 & 4237.35 & 0.23 & -24.66 & $<\mathbf{0 . 0 1}$ \\
\hline
\end{tabular}




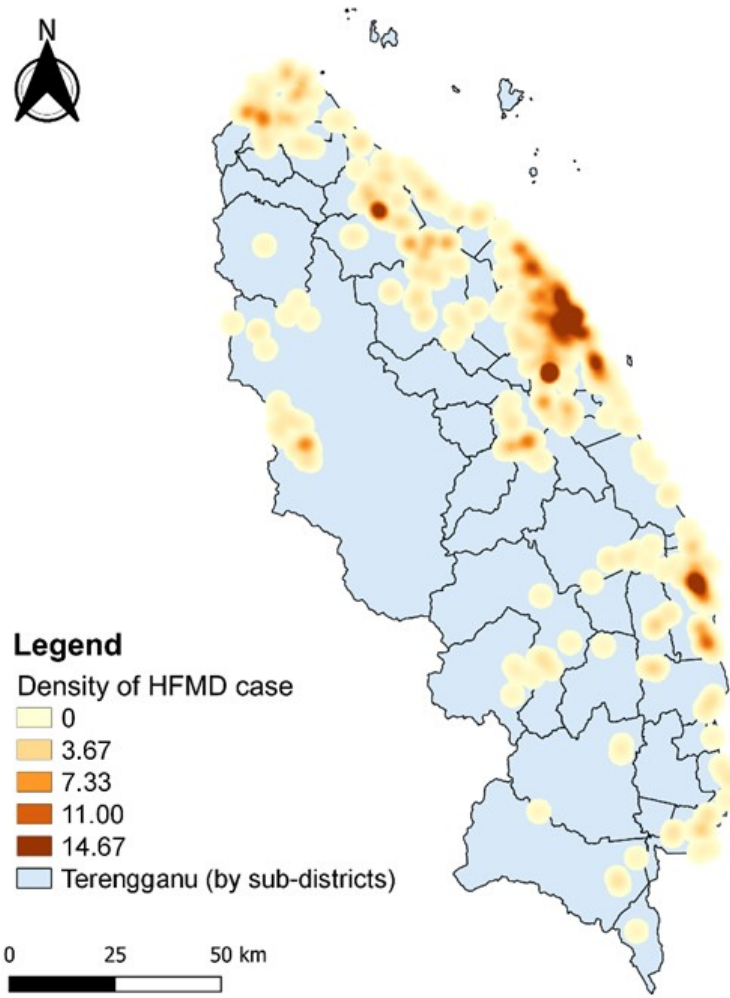

Figure 4: Geographical Density of HFMD cases in Terengganu

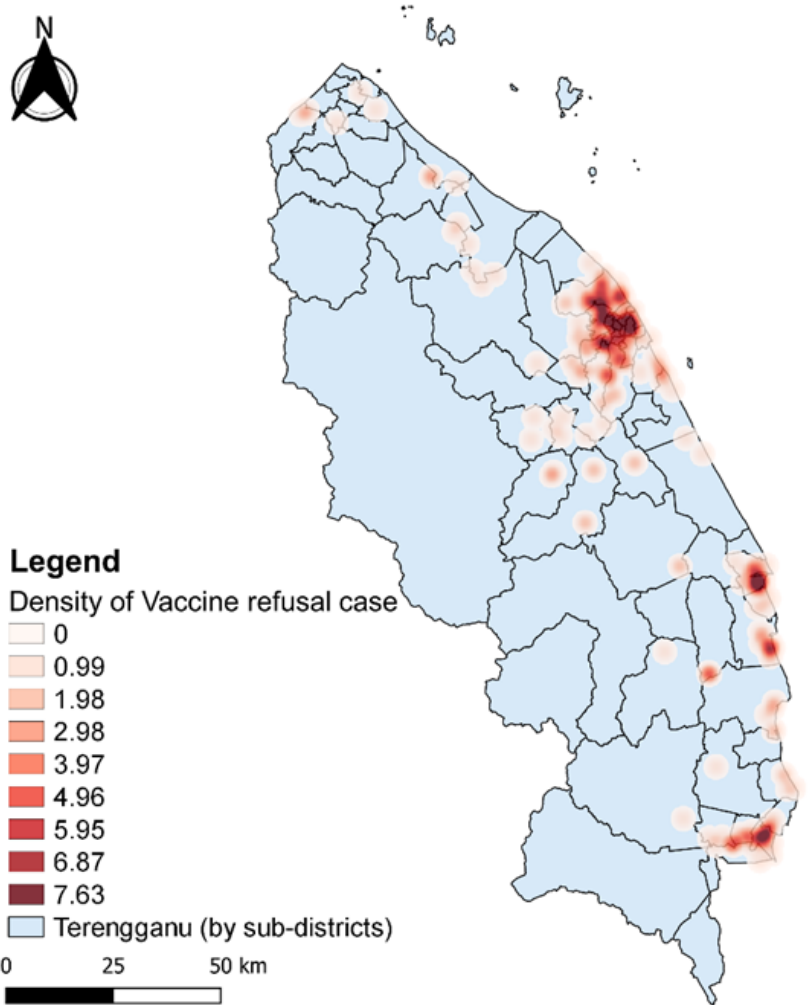

Figure 5: Geographical density of vaccine refusal cases in Terengganu 


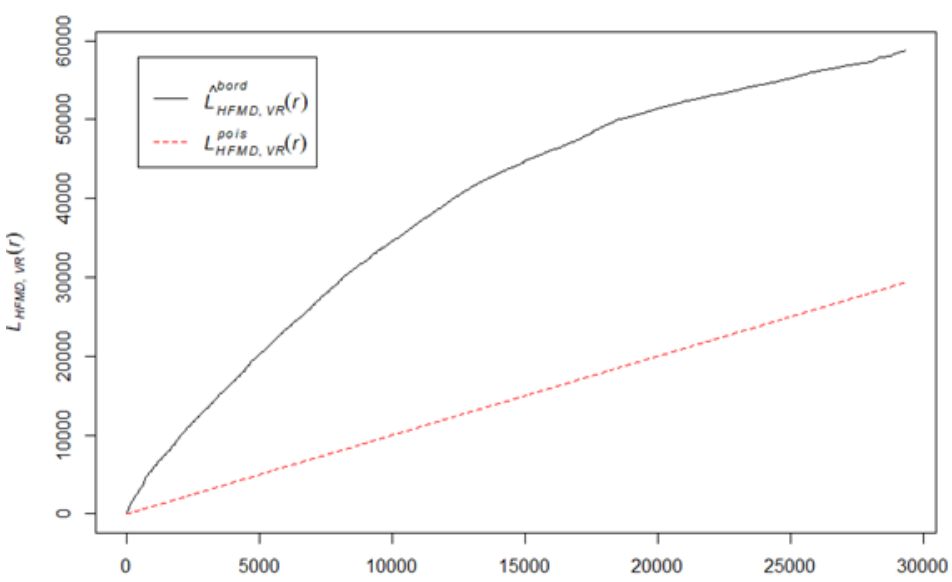

Figure 6: Cross K-Function of HFMD and vaccine refusal cases

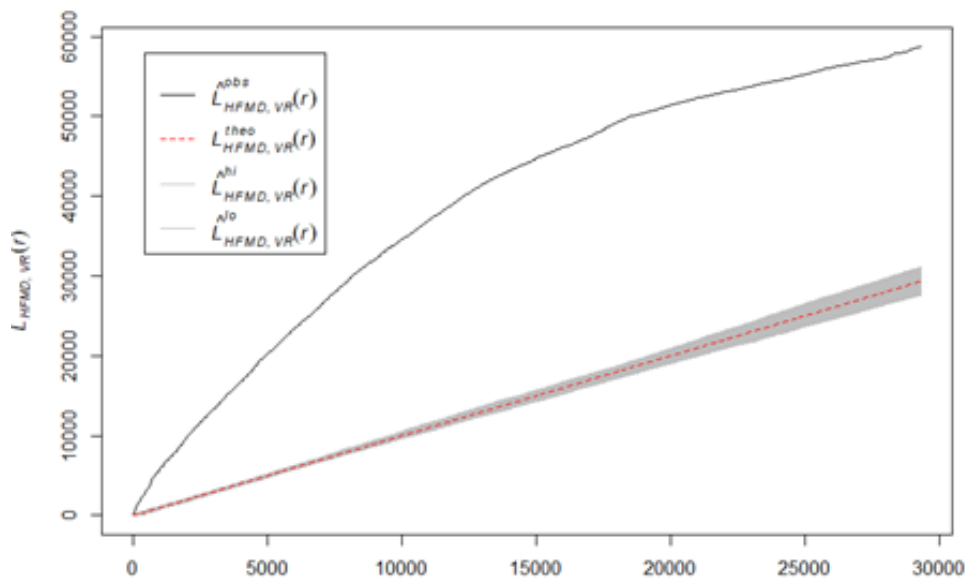

Figure 7: Envelope Cross K-Function of HFMD and vaccine refusal cases (using Monte Carlo simulation)

\subsection{The Density of HFMD and Vaccine Refusal Cases in Terengganu}

The heat map analysis of HFMD and vaccine refusal cases are shown in Figure 4 and Figure 5, respectively. Dark brown and red areas in Figure 4 and Figure 5 showed the highest density and hot spot areas of cases per three-kilometer radius, followed by lighter colours. There were three prominent hotspots of HFMD cases in Terengganu, specifically in Kuala Terengganu, Marang and Dungun.

\subsection{The Spatial Relationship between HFMD Cases and Vaccine Refusal Cases}

The observed cross Ripley's K-function (straight line) and the theoretical Poisson K-function (red dashed line) shown in Figure 6 demonstrated that both the HFMD and vaccine refusal cases were clustered together. An observed cross K-curve was plotted well above the theoretical K-curve, indicating that HFMD cases cluster around vaccine refusal cases. The Monte Carlo simulation of random labelling of events is illustrated in Figure 7. The observed cross K-function was in a straight curve, and the grey zone area represented the confidence envelope. The cross K-function (between HFMD and vaccine refusal cases) revealed an interaction between both cases as the observed curve is above the Poisson curve (what would be expected under randomness). It means that the HFMD cases stayed closely to vaccine refusal cases under the complete spatial randomness. Hence, the hypothesis of complete spatial randomness of HFMD and vaccine refusal cases was rejected. Data also revealed significant clustering evidence of vaccine refusal cases around the HFMD cases.

\section{Discussion}

The overall prevalence of HFMD in Terengganu showed a $17.3 \%$ increment in cases compared to the previous year (2015), which recorded 68.4 per 100,000 population. However, our estimate was lower than the 2016 national estimate at 148.5 cases per 100,000 population $(\mathrm{MOH}, 2018)$. 
Furthermore, the sex-specific prevalence in our study is similar to findings reported by prior studies (Ang et al., 2009 and Wang et al., 2013). However, some areas remain unclear whether the susceptibility attributes to the sex-related difference in HFMD specifically at the host genetic level (Chang et al., 2012). The role of sex hormones in regulating the immune system (Falagas et al., 2007), and the sociocultural value where parents were paying more attention to a male child than a female child (Wang et al., 2013).

This study revealed that HFMD cases predominantly infected children aged below five years, with cases from the one-year-old age group having the highest age-specific prevalence. HFMD prevalence decreased with increasing age.

These findings are consistent with those reporting that children less than five years had the highest risk for HFMD infection (Hooi et al., 2002, Ang et al., 2009, NikNadia et al., 2016 and Huang et al., 2018). This could be due to several factors include the decline of the maternally derived antibodies leading to a lack of protective immunity and an increasing number of childcare centres in Malaysia (including Terengganu), as documented in the Malaysian Early Childhood Care and Education Policy Review 2008. It was reported that the number of pre-school centers increased from $17 \%$ to $29 \%$ for each year from 2003 to 2009 (Curriculum Development Center Ministry of Education Malaysia, 2008). As a result, more children congregate inside limited spaces, providing environments with a higher risk of rapid circulation of the virus along with a higher risk of transmission to their family members and the rest of the population (Deng et al., 2011).

This study showed that HFMD cases were primarily concentrated in each district's central region (urban areas) in Terengganu, except for Besut and Kemaman districts. This finding aligned with the previous study conducted in Sarawak, wherein most HFMD cases stayed in more urban divisions (Kuching, Sibu, and Miri) than the rural divisions (Noraishah and Krishnarajah, 2016). The apparent high density of HFMD cases in towns seems to be attributed to the nature of disease transmission, in which HFMD spread mainly through the faecal-oral route and respiratory droplets; body excreta including saliva, sputum, nasal discharge, and faeces (Chang et al., 2004). The transmission rate increases in crowded and high concentration populations, especially involving infants and children in school and childcare centres (Hu et al., 2012). A study conducted in China reported that child-population density alone could explain $56 \%$ of the variance of HFMD incidence compared to climate factors which explained about 52\% (Hu et al., 2012). Therefore, for HFMD disease prevention and control, children, parents, health care workers shall be particular about hand hygiene and regular sanitation of the premises (Chang et al., 2011).

Besides, the vaccine refusal cases in Terengganu in 2016 were closely distributed to the HFMD cases. The cases were mainly concentrated in the highdensity population areas such as urban areas. Furthermore, parents who refused vaccines were mostly from Malay ethnic groups (Lim et al., 2016). Most of them came from the low socio-economic group, having secondary-level education, and did not trust vaccines as protective (Lim et al., 2016 and Ahmad et al., 2017).

A study conducted in Michigan showed the clustering of non-medical exemption to immunisation was associated with high population density, the proportion of racial/ethnic minorities in a census tract, the proportion of children aged less than five years, and mean family size (Omer et al., 2008). At the same time, other literature suggested that the cause for vaccination refusal depends on multiple factors, including cultural, emotional, religious, and social issues (Barbacariu, 2014).

Current findings highlighted the threat from inadequate immunisation coverage in Malaysia (Lim et al., 2017) and the need for a national electronic database that records childhood immunisation to facilitate vaccination tracking (Ahmed et al., 2018). This study has been used the use of state-wide data and with the application of extensive spatial analysis on HFMD and vaccine refusal cases. Collectively, our results appeared consistent with other international studies (Ang et al., 2009, Wang et al., 2013, NikNadia et al., 2016 and Huang et al., 2018).

However, the extent to which vaccine refusal contributing to HFMD infections is unknown. It is essential to highlight that vaccine refusal events were proven to cause the vaccine-preventable disease cluster and exposed the young children at increased risk for illness and death related to infectious disease (Omer et al., 2009). The application of spatio-temporal analysis and GIS in investigating communicable diseases has provided additional insight for public health physicians to formulate effective strategies in the prevention and control of HFMD. They can do that by considering these parameters: 1) density of population, 2) identification of a disease cluster, 3) the number of child care centres, in particular those overcrowded ones, and 4) presence of vaccine refusal events in that area (Safian et al., 2008 and Samphutthanon et al., 2014). 


\section{Conclusion}

HFMD cases were geographically spread over the Terengganu region and primarily concentrated in the high-density population areas, such as Kuala Terengganu, Marang, and Dungun. The locations of HFMD cases in Terengganu were statistically related to the areas of the vaccine refusal cases. These findings are valuable in assisting the public health personnel in controlling and preventing infectious disease and vaccine-related disease.

\section{Acknowledgement}

The authors would like to acknowledge the Director General of Health Malaysia for his permission to publish this paper and thank everyone who has contributed to this study.

\section{References}

Ahmad, N., Jahis, R., Kuay, L., Jamaluddin, R. and Aris, T., 2017, Primary Immunization among Children in Malaysia: Reasons for Incomplete Vaccination. J Vaccines Vaccin, Vol. 8(3), 1-8, DOI:10.4172/2157-7560.1000358.

Ahmed, A., Lee, K. S., Bukhsh, A., Al-Worafi, Y. M., Sarker, M. M. R., Ming, L. C. and Khan, T. M., 2018, Outbreak of Vaccine-Preventable Diseases in Muslim Majority Countries. Journal of Infection and Public Health, Vol. 11(2), 153155.

Ang, L. W., Koh, B. K., Chan, K. P., Chua, L. T., James, L. and Goh, K. T., 2009, Epidemiology and Control of Hand, Foot and Mouth Disease in Singapore. Ann Acad Med Singapore, Vol. 38(2), 106-112.

Baddeley, A. and Turner, R., 2005, Spatstat: an R Package for Analyzing Spatial Point Patterns. Journal of Statistical Software, Vol. 12(1), 1-42.

Barbacariu, C. L., 2014, Parents' Refusal to Vaccinate their Children: An Increasing Social Phenomenon which Threatens Public Health. Procedia-Social and Behavioral Sciences, Vol. 149, 84-91.

Brinkhoff, T., 2018, Terengganu. Retrieved from: https://www.citypopulation.de/php/malaysiaadm in.php?admlid=11 [Accessed 22 November].

Chang, A. C., Jacobsen, K. H., Lin, K. W. and Teng, L. J., 2011, Enterovirus Knowledge and Hand Washing Practices among Nurses in a Hospital in Taipei, Taiwan. Taiwan Epidemiology Bulletin, Vol. 27(6), 81-101.
Chang, H. L., Chio, C. P., Su, H. J., Liao, C. M., Lin, C. Y., Shau, W. Y., Chi, Y. C., Cheng, Y. T., Chou, Y. L. and Li, C. Y., 2012, The Association between Enterovirus 71 Infections and Meteorological Parameters in Taiwan. PLoS $O N E$, Vol. 7(10), 1-5, DOI: 10.1371/journal.pone.0046845.

Chang, L. Y., Tsao, K. C., Hsia, S. H., Shih, S. R., Huang, C. G., Chan, W. K., Hsu, K. H., Fang, T. Y., Huang, Y. C. and Lin, T. Y., 2004, Transmission and Clinical Features of Enterovirus 71 Infections in Household Contacts in Taiwan. Jama, Vol. 291(2), 222-227.

Charoenchokpanit, R. and Pumpaibool, T., 2013, Knowledge Attitude and Preventive Behaviors Towards Hand Foot and Mouth Disease among Caregivers of Children Under Five Years old in Bangkok, Thailand. Journal of Health Research, Vol. 27(5), 281-286.

Core, R. T. and Team, R., 2013, R: A Language and Environment for Statistical Computing. Vienna, Austria: R Foundation for Statistical Computing. DOI:10.1890/0012-9658(2002)083[3097:CFHIWS]2.0.CO;2.

Deng, C., Yang, C., Wan, J., Zhu, L. and Leng, Q., 2011, Irregular Poliovirus Vaccination Correlates to Pulmonary Edema of Hand, Foot, and Mouth Disease. Clinical and Vaccine Immunology, Vol. 18(9), 1589-1590.

DOSM. 2019, Terengganu. Retrieved from:https://www.dosm.gov.my/v1/index.php?r $=$ column/cone\&menu_id=ZnlmM1RFWjNDZGt 5ZnBrbkpNNkkxUT09 [Accessed 22 November].

Falagas, M. E., Mourtzoukou, E. G. and Vardakas, K. Z., 2007, Sex Differences in the Incidence and Severity of Respiratory Tract Infections. Respiratory medicine, Vol. 101(9), 1845-1863.

Hooi, P., Chua, B., Lee, C., Lam, S. and Chua, K., 2002, Hand, Foot and Mouth Disease: University Malaya Medical Centre Experience. The Medical Journal of Malaysia, Vol. 57(1), 88-91.

Hu, M., Li, Z., Wang, J., Jia, L., Liao, Y., Lai, S., Guo, Y., Zhao, D. and Yang, W., 2012, Determinants of the Incidence of Hand, Foot and Mouth Disease in China using Geographically Weighted Regression Models. PloS one, Vol. 7(6), 1-8, DOI:10.1371/journal.pone.0038978.

Huang, J., Liao, Q., Ooi, M. H., Cowling, B. J., Chang, Z., Wu, P., Liu, F., Li, Y., Luo, L. and Yu, S., 2018, Epidemiology of Recurrent Hand, Foot and Mouth Disease, China, 2008-2015. Emerging Infectious Diseases, Vol. 24(3), 432. 
Huang, J., Wang, J., Bo, Y., Xu, C., Hu, M. and Huang, D., 2014, Identification of Health Risks Of Hand, Foot and Mouth Disease in China Using the Geographical Detector Technique. International Journal of Environmental Research and Public Health, Vol. 11(3), 34073423.

Lai, P. C., So, F. M. and Chan, K. W., 2008, Spatial Epidemiological Approaches in Disease Mapping and Analysis: CRC Press. DOI:10.1201/9781420045536.

Lawson, A. B., 2018, Bayesian Disease Mapping: Hierarchical Modeling in Spatial Epidemiology: Chapman and Hall/CRC. DOI:10.1201/9781584888413.

Lim, K., Chan, Y., Ani, A. N., Rohani, J., Norfadhilah, Z. S. and Santhi, M., 2017, Complete Immunization Coverage and its Determinants among Children in Malaysia: Findings from the National Health and Morbidity Survey (NHMS) 2016. Public health, Vol. 153, 52-57.

Lim, W., Amar S., H., Jeganathan, N., Rahmat, H., Mustafa, N., Mohd Yusof, F. S., Rahman, R., Itam, S., Chan, C. and Julia, M., 2016, Exploring Immunisation Refusal by Parents in the Malaysian Context. Cogent Medicine, Vol. 3(1), 1-6, DOI:10.1080/2331205X.2016.1142410.

MOH, 2016, Health Facts 2001-2010. Retrieved from:http://vlib.moh.gov.my/cms/content.jsp?id=com.tms.cms.section.Section_2e86ac 04a0188549-d5315d00-8f1faeb6 [Accessed 12 March].

MOH, 2018, Main MOH Publications; Health Facts 2016. . Retrieved from:http://www.moh.gov.my/english.php/pages/view/56 [Accessed 12 March].

NikNadia, N., Sam, I. C., Rampal, S., WanNorAmalina, W., NurAtifah, G., Verasahib, K., Ong, C. C., Mohd Adib, M. and Chan, Y. F., 2016, Cyclical Patterns of Hand, Foot and Mouth Disease Caused by Enterovirus A71 in Malaysia. PLoS Neglected Tropical Diseases, Vol. 10(3), https://doi.org/10.1371/journal.pntd.0004562.

Noraishah, M. and Krishnarajah, I., 2016, Use of GIS Mapping for HFMD Cases in Sarawak, Malaysia. International Journal, Vol. 5(10), 1937-1945.
Omer, S. B., Enger, K. S., Moulton, L. H., Halsey, N. A., Stokley, S. and Salmon, D. A., 2008, Geographic Clustering of Nonmedical Exemptions to School Immunization Requirements and Associations with Geographic Clustering of Pertussis. American Journal of Epidemiology, Vol. 168(12), 1389-1396.

Omer, S. B., Salmon, D. A., Orenstein, W. A., Dehart, M. P. and Halsey, N., 2009, Vaccine Refusal, Mandatory Immunization, and the Risks of Vaccine-Preventable Diseases. New England Journal of Medicine, Vol. 360(19), 1981-1988.

Safian, N., Shah, S. A., Idrus, S. and Hamzah, W. M., 2008, Cluster Analysis of Typhoid Cases in Kota Bharu, Kelantan, Malaysia. Medical Journal of Indonesia, Vol. 17(3), 175-182.

Samphutthanon, R., Tripathi, N. K., Ninsawat, S. and Duboz, R., 2014, Spatio-Temporal Distribution and Hotspots of Hand, Foot and Mouth Disease (HFMD) in Northern Thailand. International Journal of Environmental Research and Public Health, Vol. 11(1), 312336.

Sham, N. M., Krishnarajah, I., Ibrahim, N. A. and Lye, M. S., 2014, Temporal and Spatial Mapping of Hand, Foot and Mouth Disease in Sarawak, Malaysia. Geospatial Health, Vol. 8(2), 503-507.

Wang, Y., Sun, L., Xiao, W., Chen, L., Wang, X. and Pan, D., 2013, Epidemiology and Clinical Characteristics of Hand Foot, and Mouth Disease in a Shenzhen Sentinel Hospital from 2009 to 2011. BMC Infectious Diseases, Vol. 13(1), 1-5.

Xing, W., Liao, Q., Viboud, C., Zhang, J., Sun, J., Wu, J. T., Chang, Z., Liu, F., Fang, V. J. and Zheng, Y., 2014, Hand, Foot, and Mouth Disease in China, 2008-12: An Epidemiological Study. The Lancet Infectious Diseases, Vol. 14(4), 308-318.

Yeap, E. and Uy, I., 2014, Marker clustering And Heatmaps: New Features in the Google Maps Android API Utility Library. Google Geo Developers. 